\title{
HIROTA DIFFERENCE EQUATION AND A COMMUTATOR IDENTITY ON AN ASSOCIATIVE ALGEBRA
}

\author{
A. K. POGREBKOV
}

To Ludwig Dmitrievich Faddeev on the occasion of his 75 th birthday

\begin{abstract}
In earlier papers of the author it was shown that some simple commutator identities on an associative algebra generate integrable nonlinear equations. Here, this observation is generalized to the case of difference nonlinear equations. The identity under study leads, under a special realization of the elements of the associative algebra, to the famous Hirota difference equation. Direct and inverse problems are considered for this equation, as well as some properties of its solutions. Finally, some other commutator identities are discussed and their relationship with integrable nonlinear equations, both differential and difference, is demonstrated.
\end{abstract}

\section{§1. Algebraic identities}

1.1. Commutator identity and a linear difference equation. Suppose we have an associative algebra with unity $I$ over the complex field $\mathbb{C}$. Let $a_{1}, a_{2}$, and $a_{3}$ be some complex parameters. Then for any pair $A, B$ of elements of this algebra such that $\left(A-a_{1} I\right)^{-1},\left(A-a_{2} I\right)^{-1}$, and $\left(A-a_{3} I\right)^{-1}$ exist in this algebra, we have the following identity:

$$
\begin{aligned}
\sum_{i, j, k=1}^{3} \varepsilon_{i j k}\left(a_{j}-a_{k}\right) & {\left[\left(A-a_{i}\right) B\left(A-a_{i}\right)^{-1}\right.} \\
+ & \left.\left(A-a_{j}\right)\left(A-a_{k}\right) B\left(A-a_{j}\right)^{-1}\left(A-a_{k}\right)^{-1}\right]=0,
\end{aligned}
$$

where $\varepsilon_{i j k}$ is a totally antisymmetric tensor, $\varepsilon_{123}=1$, and where we have omitted the unity operator as a multiplier of $a_{j}$. This identity is a trivial consequence of associativity, and it is proved by multiplying, say, from the right by $\left(A-a_{1}\right)\left(A-a_{2}\right)\left(A-a_{3}\right)$. One can consider a more general situation where the $a_{j}$ are elements of the same algebra that commute with $A$ and $B$, but here we use $a_{j} \in \mathbb{C}$. Relation (1.1) can be written in a simpler form if we introduce the operation

$$
\delta_{a}(B)=(A-a) B(A-a)^{-1}-B
$$

on the algebra. Then (1.1) reduces to

$$
\left(a_{1}-a_{2}\right) \delta_{a_{1}} \delta_{a_{2}}+\left(a_{2}-a_{3}\right) \delta_{a_{2}} \delta_{a_{3}}+\left(a_{3}-a_{1}\right) \delta_{a_{3}} \delta_{a_{1}}=0
$$

where the element $B$ is omitted and $\left(\delta_{a_{1}} \delta_{a_{2}}\right)(B) \equiv \delta_{a_{1}}\left(\delta_{a_{2}}(B)\right)$, etc. The operations $\delta_{a_{1}}$, $\delta_{a_{2}}$, and $\delta_{a_{3}}$ are close to the commutator in the sense of groups, where the first term in (1.2) is denoted by ${ }^{(A-a)} B$. It is clear that the algebra generated by these operations

2010 Mathematics Subject Classification. Primary 37K15.

Key words and phrases. Hirota difference equation, commutator identity, extended operators, direct and inverse problems.

Supported in part by RFBR (grants 09-01-12150 and 09-01-93106), by Scientific Schools Program, and by the RAS program "Fundamental Problems of Nonlinear Dynamics". 
is commutative, but it is not free, because relation (1.3) is a constraint in the sense of this algebra.

Now we introduce dependence on discrete "times" $m_{n} \in \mathbb{Z}, n=1,2,3$,

$$
B\left(m_{1}, m_{2}, m_{3}\right)=\left(\prod_{n=1}^{3}\left(A-a_{n}\right)^{m_{n}}\right) B\left(\prod_{n=1}^{3}\left(A-a_{n}\right)^{m_{n}}\right)^{-1} .
$$

In what follows we denote $B(m)=B\left(m_{1}, m_{2}, m_{3}\right)$ for brevity; introducing the difference operator by

$$
\Delta_{n} B(m)=B\left(m+e_{n}\right)-B(m), \quad n=1,2,3,
$$

where the $e_{n}$ are the "vectors"

$$
e_{1}=(1,0,0), \quad e_{2}=(0,1,0), \quad e_{3}=(0,0,1)
$$

we see that

$$
\left(\delta_{a_{n}}(B)\right)(m)=\Delta_{n} B(m) .
$$

Thus, by (1.1) (or equivalently, (1.3) $), B(m)$ obeys the following difference equation:

$$
\left[\left(a_{1}-a_{2}\right) \Delta_{1} \Delta_{2}+\left(a_{2}-a_{3}\right) \Delta_{2} \Delta_{3}+\left(a_{3}-a_{1}\right) \Delta_{3} \Delta_{1}\right] B(m)=0 .
$$

Below we develop a procedure that enables derivation of the nonlinear difference equation (specifically, the Hirota equation) and its Lax pair via a special realization of the elements $A$ and $B$. In this construction equation (1.8) will play the role of the equation on the spectral data of the Lax operator. Here we only mention that if $a_{1}=a_{2}=a_{3}$, then equation (1.8) becomes meaningless. If, say, $a_{1}=a_{2} \neq a_{3}$, the equation is trivially solvable: $B(m)=B_{1}\left(m_{1}, m_{2}\right)+B_{2}\left(m_{1}+m_{2}, m_{3}\right)$, where $B_{1}$ and $B_{2}$ are arbitrary functions of their arguments. We see that the Cauchy problem with respect to one of the variables $m_{n}$ is undetermined in this case. In order to avoid such degenerate situations, we always assume that all three parameters $a_{n}$ are different.

\section{§2. Hirota Difference EQUATion, AND THE LAX PAIR}

2.1. Extended operators. The function $B(\mathrm{~m})$ can be viewed as a function of two "space" variables (say, $m_{1}$ and $m_{2}$ ) and one "time" variable (correspondingly, $m_{3}$ ) with evolution given by (1.8). Thus, here we deal with functions of two variables and their evolution with respect to the third variable. The operators on this set of functions are (infinite) matrices $\mathcal{F}_{m, m^{\prime}}$, where $m$ and $m^{\prime}$ are double indices, $m=\left(m_{1}, m_{2}\right), m^{\prime}=$ $\left(m_{1}^{\prime}, m_{2}^{\prime}\right)$, and $m_{1}, m_{2}, m_{1}^{\prime}, m_{2}^{\prime} \in \mathbb{Z}$. In order to develop our construction, we embed a subspace of these matrices in the set of some special objects; by analogy with the continuous case, these objects will be called extended operators; see 3 - 6 .

Let $F\left(\zeta_{1}, \zeta_{2} ; z_{1}, z_{2}\right) \equiv F(\zeta ; z)$ be a distribution,

$$
F\left(\zeta_{1}, \zeta_{2} ; z_{1}, z_{2}\right) \in \mathcal{S}^{\prime}
$$

with respect to the variables

$$
\zeta=\left(\zeta_{1}, \zeta_{2}\right), \quad z=\left(z_{1}, z_{2}\right), \quad z_{j}, \zeta_{j} \in \mathbb{C}, \quad\left|\zeta_{j}\right|=1, \quad j=1,2 .
$$

We emphasize that no kind of analyticity of the functions $F\left(\zeta_{1}, \zeta_{2} ; z_{1}, z_{2}\right)$ with respect to their variables is assumed, and we omit writing, say, the variables $\bar{z}$ in the dependence of $F$ only for brevity. For the same reason, in what follows we use expressions of the kind $z \zeta, 1 / z$, etc., to denote the following pairs of complex variables:

$$
z \zeta=\left(z_{1} \zeta_{1}, z_{2} \zeta_{2}\right), \quad 1 / z=\left(1 / z_{1}, 1 / z_{2}\right) .
$$


On the set of such functions $F(\zeta ; z)$, we define the following linear operations:

$$
\begin{array}{ll}
\text { transposition: } & F^{\mathrm{T}}(\zeta ; z)=F\left(\zeta ;(z \zeta)^{-1}\right), \\
\text { complex conjugation: } & F^{*}(\zeta ; z)=\overline{F(\bar{\zeta} ; \bar{z}),}
\end{array}
$$

and

$$
\text { Hermitian conjugation: } \quad F^{\dagger}(\zeta ; z)=\overline{F(\bar{\zeta} ; \zeta / \bar{z})},
$$

so that we have $F^{\dagger}=\left(F^{\mathrm{T}}\right)^{*}$, as always. We also define the composition of such functions via the integral

$$
(F G)(\zeta ; z)=\oint_{\left|\zeta^{\prime}\right|=1}\left(\frac{d \zeta^{\prime}}{2 \pi i \zeta^{\prime}}\right)^{2} F\left(\zeta \bar{\zeta}^{\prime} ; z \zeta^{\prime}\right) G\left(\zeta^{\prime} ; z\right)
$$

if it exists in the sense of distributions; here we have used the notation

$$
\oint_{|\zeta|=1}\left(\frac{d \zeta}{2 \pi i \zeta}\right)^{2} \cdots \equiv \oint_{\left|\zeta_{1}\right|=1} \frac{d \zeta_{1}}{2 \pi i \zeta_{1}} \oint_{\left|\zeta_{2}\right|=1} \frac{d \zeta_{2}}{2 \pi i \zeta_{2}} \cdots
$$

Let $\mathcal{F}_{m, m^{\prime}}$ be an arbitrary matrix such that the "Fourier series"

$$
F(\zeta ; z)=\sum_{m, m^{\prime} \in \mathbb{Z}^{2}} z_{1}^{m_{1}^{\prime}-m_{1}} z_{2}^{m_{2}^{\prime}-m_{2}} \zeta_{1}^{-m_{1}} \zeta_{2}^{-m_{2}} \mathcal{F}_{m, m^{\prime}}
$$

converges in the sense of distribution (see (2.1)), where the variables $\zeta$ and $z$ obey conditions (2.2). Then this relation determines a map

$$
\mathcal{F}_{m, m^{\prime}} \rightarrow F\left(\zeta_{1}, \zeta_{2} ; z_{1}, z_{2}\right)=F(\zeta ; z),
$$

i.e., the embedding mentioned above. It is clear that by (2.9) we get a function depending on six real variables, instead of a matrix depending on four real numbers. For this reason, all operators $F$ introduced above will be called extended operators with kernels $F(\zeta ; z)$. Now, defining

$$
\mathcal{F}_{m, m^{\prime}}(|z|)=\oint_{|\zeta|=1}\left(\frac{d \zeta}{2 \pi i \zeta}\right)^{2} \zeta_{1}^{m_{1}} \zeta_{2}^{m_{2}} \oint_{\left|z^{\prime}\right|=|z|}\left(\frac{d z^{\prime}}{2 \pi i z^{\prime}}\right)^{2} z_{1}^{\prime m_{1}-m_{1}^{\prime}} z_{2}^{\prime m_{2}-m_{2}^{\prime}} F\left(\zeta ; z^{\prime}\right)
$$

we easily see that if $F(\zeta ; z)$ (see (2.9)) is the image of a matrix $\mathcal{F}_{m, m^{\prime}}$, then the right-hand side is independent of $\left|z_{1}\right|$ and $\left|z_{2}\right|$, and this relation is the inversion of the transformation (2.9). In the case where $F$ extends a matrix $\mathcal{F}$, it is easy to check that $F^{\mathrm{T}}, F^{*}$, and $F^{\dagger}$ in (2.4)-(2.6) are the kernels of the transposed, complex conjugate, and Hermitian conjugate matrices $\mathcal{F}^{\mathrm{T}}, \mathcal{F}^{*}$, and $\mathcal{F}^{\dagger}$. In the same way we can check that in this case the standard product of matrices,

$$
(\mathcal{F} \mathcal{G})_{m, m^{\prime}}=\sum_{n=\left(n_{1}, n_{2}\right)} \mathcal{F}_{m, n} \mathcal{G}_{n, m^{\prime}}
$$

has extension $F G$ with kernel given by (2.7).

As the simplest examples of extensions, we consider the unity and shift matrices. The image of the unity matrix is the unity (with respect to composition (2.7)) operator $I$ :

$$
\mathcal{I}_{m, m^{\prime}}=\delta_{m_{1}, m_{1}^{\prime}} \delta_{m_{2}, m_{2}^{\prime}} \mapsto I(\zeta ; z)=\delta_{c}(\zeta) \equiv \delta_{c}\left(\zeta_{1}\right) \delta_{c}\left(\zeta_{2}\right),
$$

where

$$
\delta_{c}\left(\zeta_{j}\right)=\sum_{n=-\infty}^{\infty} \zeta_{j}^{n}
$$


is the delta-function on the contour, i.e.,

$$
\oint_{\left|\zeta_{1}\right|=1} \frac{d \zeta_{1}}{2 \pi i \zeta_{1}} f\left(\zeta_{1}\right) \delta_{c}\left(\zeta_{1}\right)=f(1)
$$

for a test function $f(\zeta)$. Likewise, for the (infinite) shift matrices $\left(\mathcal{T}_{1}\right)_{m, m^{\prime}}$ and $\left(\mathcal{T}_{2}\right)_{m, m^{\prime}}$ we have

$$
\begin{array}{lll}
\left(\mathcal{T}_{1}\right)_{m, m^{\prime}}=\delta_{m_{1}+1, m_{1}^{\prime}} \delta_{m_{2}, m_{2}^{\prime}} & \mapsto \quad T_{1}(\zeta ; z)=z_{1} \delta_{c}(\zeta), \\
\left(\mathcal{T}_{2}\right)_{m, m^{\prime}}=\delta_{m_{1}, m_{1}^{\prime}} \delta_{m_{2}+1, m_{2}^{\prime}} & \mapsto \quad T_{2}(\zeta ; z)=z_{2} \delta_{c}(\zeta) .
\end{array}
$$

Clearly, a characteristic property of the quasidiagonal matrices is that the kernels of their extensions (2.9) are meromorphic functions of the variables $z_{1}$ and $z_{2}$ (with the only singularity at zero).

As was mentioned before, in a generic situation the functions $F(\zeta ; z)$ have no property of analyticity. The main advantage of the set of extended operators is the possibility to associate, with any operator $F$, two new operators $\bar{\partial}_{1} F$ and $\bar{\partial}_{2} F$ with kernels given in terms of the $\bar{\partial}$-derivatives with respect to $z_{j}$ :

$$
\left(\bar{\partial}_{j} F\right)(\zeta ; z)=\frac{\partial F(\zeta, z)}{\partial \bar{z}_{j}}, \quad j=1,2,
$$

so that they control the deviation of $F$ from analyticity. By (2.7), the Leibniz rule for these $\bar{\partial}$-derivatives takes the form

$$
\bar{\partial}_{j}(F G)=\left(\bar{\partial}_{j} F\right) T_{j}^{-1} G T_{j}+F \bar{\partial}_{j} G .
$$

For future references, we mention that in the specific case where the kernel of $F$ equals $F(\zeta ; z)=f(z) \delta_{c}(\zeta)$, from (2.7) and (2.14) it follows that the similarity transformation of an arbitrary operator $G$ has the kernel

$$
\left(F G F^{-1}\right)(\zeta ; z)=\frac{f(\zeta z)}{f(z)} G(\zeta ; z) .
$$

2.2. Operator realization of elements of an associative algebra. The operator $L_{0}$. We realize elements $A$ and $B$ of an associative algebra as extended operators in the sense of the definitions in Subsection 2.1 with kernels $A(\zeta ; z)$ and $B(\zeta ; z)$, respectively. Here we impose conditions on this realization that enable us to derive the Lax pair and nonlinear integrable equations; see 1 and 2 .

Condition 1. Introducing the "time" dependence on $m_{1}, m_{2}$, and $m_{3}$ via relation (1.4) gives an operator $B(m)$ with kernels $B(m, \zeta ; z)$ belonging to the same space of operators.

By (1.4) and (1.6), we have

$$
B\left(m+e_{n}\right)=\left(A-a_{n}\right) B(m)\left(A-a_{n}\right)^{-1}, \quad n=1,2,3 .
$$

In order to use this property, we recall that in the algebraic approach to integrable hierarchies (see [7- 9]) it was proved that under the Zakharov-Shabat procedure [10, the two lowest times simply shift the space variables (in the case of the main symmetries, [11]). Under our approach, choosing $m_{1}$ and $m_{2}$ to be space variables as in Subsection 2.1, we impose the following condition.

Condition 2. The dependence of $B(m, \zeta ; z)$ on $m_{1}$ and $m_{2}$ reduces to a shift of the matrix variables; in terms of the shift operators (2.15), this is equivalent to the relations

$$
B\left(m+e_{j}\right)=T_{j} B(m) T_{j}^{-1}, \quad j=1,2,
$$

for an arbitrary operator $B(m)$. We emphasize that, in contrast to (2.19), this condition is imposed on the space variables $m_{1}$ and $m_{2}$ only, and not on the time variable $m_{3}$. 
Because of (2.15) and (2.18), this condition means that the kernel of the operator $B$ has the following representation:

$$
B\left(m_{1}, m_{2}, m_{3}, \zeta ; z\right)=\zeta^{m_{1}} \zeta^{m_{2}} B\left(m_{3}, \zeta ; z\right),
$$

where $B\left(m_{3}, \zeta ; z\right)$ is a function of its arguments, i.e., a function independent of $m_{1}$ and $m_{2}$. Then condition (2.19) with $n=1$ shows that we can choose

$$
A=T_{1}+a_{1}, \quad \text { i.e., } \quad A(\zeta ; z)=\left(z_{1}+a_{1}\right) \delta_{c}(\zeta),
$$

where we have used (2.12) and (2.15). In general, $A-a_{1}$ can be chosen proportional to $T_{1}$ with a factor commuting with $B$. But, by (2.15), such a coefficient is already included in $T_{1}$ : this is $\left|z_{1}\right|$, which, by (2.18), commutes with any operator. Since $B$ is arbitrary, we see that the choice in (2.22) is unique.

Now, by (1.4), condition (2.19) with $n=2$ takes the form

$$
B\left(m_{2}+1\right)=T_{2} B(m) T_{2}^{-1}=\left(T_{1}+\left(a_{1}-a_{2}\right) I\right) B(m)\left(T_{1}+\left(a_{1}-a_{2}\right) I\right)^{-1},
$$

i.e., there exists an operator

$$
L_{0}=T_{2}-T_{1}+\left(a_{2}-a_{1}\right) I
$$

such that

$$
L_{0} B=T_{2} B T_{2}^{-1} L_{0} .
$$

Relation (2.22) shows that the kernel of the operator $A$ is independent of $z_{2}$. Thus, as in [1, we also impose the next condition.

\section{Condition 3.}

$$
B(\zeta ; z)=B\left(\zeta ; z_{1}\right)
$$

By (2.19) and (2.22), this implies also that $B(m, \zeta ; z)=B\left(m, \zeta ; z_{1}\right)$.

Summarizing, we can write (2.25) as an equation for the kernel $B(\zeta ; z)$ :

$$
\left[z_{1}\left(\zeta_{1}-\zeta_{2}\right)+\left(1-\zeta_{2}\right)\left(a_{1}-a_{2}\right)\right] B\left(\zeta ; z_{1}\right)=0,
$$

which means that $B\left(\zeta ; z_{1}\right) \sim \delta\left(z_{1}\left(\zeta_{1}-\zeta_{2}\right)+\left(1-\zeta_{2}\right)\left(a_{1}-a_{2}\right)\right)$. Here $\delta(w)=\delta\left(w_{\Re}\right) \delta\left(w_{\Im}\right)$ is the standard $\delta$-function of the complex variable $w=w_{\Re}+i w_{\Im}$ (not the contour one defined in (2.13) $)$ 1 Thus, there exists a function $b\left(m, z_{1}\right)$ such that

$$
B\left(m, \zeta ; z_{1}\right)=\delta\left(z_{1}-\left(a_{1}-a_{2}\right) \frac{\zeta_{2}-1}{\zeta_{1}-\zeta_{2}}\right) b(m, \zeta),
$$

where, thanks to (1.4) and the $\delta$-function in (2.28), the $m$-dependence of the function $b(m, \zeta)$ looks like this:

$$
b(m, \zeta)=\zeta_{1}^{m_{1}+m_{3}} \zeta_{2}^{m_{2}+m_{3}}\left(-\frac{a_{1}-a_{2}+\left(a_{2}-a_{3}\right) \bar{\zeta}_{2}+\left(a_{3}-a_{1}\right) \bar{\zeta}_{1}}{a_{1}-a_{2}+\left(a_{2}-a_{3}\right) \zeta_{2}+\left(a_{3}-a_{1}\right) \zeta_{1}}\right)^{m_{3}} b(\zeta),
$$

$b(\zeta)$ being a function of $\zeta$ only. It is easily seen that, in general, the $m_{3}$-dependent factor may grow with $m_{3}$. In order to exclude this growth and also to preserve symmetry with respect to $m_{1}, m_{2}, m_{3}$, we must assume that this factor is unimodular. This condition, which we always assume below, is equivalent to the property that any two of the three differences $a_{1}-a_{2}, a_{2}-a_{3}, a_{3}-a_{1}$ have arguments equal $\bmod \pi$.

\footnotetext{
${ }^{1}$ Here we skip the consideration of the general situation where $B(\zeta ; z)$ is proportional to a sum of terms that involve finite derivatives of the $\delta$-function with respect to $\bar{z}_{1}$.
} 
2.3. Transformation operator, the Lax pair, and the nonlinear equation. Following the general method suggested in [1] and [2], we introduce the dressing operator $\nu$ as a solution of the following $\bar{\partial}$-equation:

$$
\bar{\partial}_{1} \nu=\nu B
$$

By analogy with Condition 3, we look for an operator whose kernel is independent of $z_{2}$, i.e., $\nu(\zeta ; z)=\nu\left(\zeta ; z_{1}\right)$. We fix a solution of (2.30) by normalizing $\nu$ to be equal the unity operator at the singularity point of $L_{0}(\zeta ; z)$ by $z_{1}$. By (2.12), (2.15), and (2.24) this kernel is equal to

$$
L_{0}(\zeta ; z)=\left(z_{2}-z_{1}+a_{2}-a_{1}\right) \delta_{c}(\zeta)
$$

and the normalization condition reads as

$$
\lim _{z_{1} \rightarrow \infty} \nu(\zeta ; z)=\delta_{c}(\zeta)
$$

In what follows we assume that problem (2.30), (2.32) is uniquely solvable.

We introduce the $m$-dependence by the equation

$$
\bar{\partial}_{1} \nu(m)=\nu(m) B(m)
$$

and preserve the same normalization condition (2.32). Then equation (2.20) results in the identities

$$
\nu\left(m+e_{1}\right)=T_{1} \nu(m) T_{1}^{-1}, \quad \nu\left(m+e_{2}\right)=T_{2} \nu(m) T_{2}^{-1} .
$$

As we have seen, the data of the $\bar{\partial}$-equation (2.30) obey (2.23). Let us consider a similar combination for the operator $\nu$. Using (2.7), (2.17), and (2.30), we get

$$
\bar{\partial}_{1}\left(L_{0} \nu-T_{2} \nu T_{2}^{-1} L_{0}\right)=\left(L_{0} \nu-T_{2} \nu T_{2}^{-1} L_{0}\right) B-T_{2} \nu T_{2}^{-1}\left(L_{0} B-T_{2} B T_{2}^{-1} L_{0}\right),
$$

where we have used the fact that $\bar{\partial}_{1} L_{0}=0$ by (2.31). Thus, by (2.23), the expression in the last parentheses equals zero, so that $L_{0} \nu-T_{2} \nu T_{2}^{-1} L_{0}$ satisfies the same equation (2.30) but with the normalization

$$
\lim _{z_{1} \rightarrow \infty}\left(L_{0} \nu-T_{2} \nu T_{2}^{-1} L_{0}\right)(\zeta ; z)=\left(\zeta_{1}-\zeta_{2}\right) U(m, \zeta),
$$

where $U(m, \zeta)$ is the $1 / z_{1}$-term of the expansion of $\nu(m, \zeta ; z)$ at $z_{1}$-infinity:

$$
\nu(m, \zeta ; z)=\delta_{c}(\zeta)+\frac{U(m, \zeta)}{z_{1}}+\cdots
$$

By the unique solvability condition for problem (2.30), (2.32), we have

$$
L \nu=T_{2} \nu T_{2}^{-1} L_{0},
$$

where

$$
L=L_{0}+T_{1} U T_{1}^{-1}-T_{2} U T_{2}^{-1},
$$

and $U(m)$ is the operator with the kernel $U(m, \zeta)$. A specific property of this kernel is its independence of $z_{1}$. Accordingly, the inverse transformation (2.10) yields

$$
\mathcal{U}_{m, m^{\prime}}=\delta_{m_{1}, m_{1}^{\prime}} \delta_{m_{2}, m_{2}^{\prime}} u(m),
$$

so that the matrix $\mathcal{U}$ is diagonal with the following diagonal elements:

$$
u(m)=\oint_{|\zeta|=1}\left(\frac{d \zeta}{2 \pi i \zeta}\right)^{2} U(m, \zeta) \equiv \oint_{|\zeta|=1}\left(\frac{d \zeta}{2 \pi i \zeta}\right)^{2} \zeta_{1}^{m_{1}} \zeta_{2}^{m_{2}} U\left(0,0, m_{3}, \zeta\right)
$$


Writing equation (2.37) as $T_{2}^{-1} L \nu=\nu T_{2}^{-1} L_{0}$, we see that the operator $\nu$ relates the "bare" operator $T_{2}^{-1} L_{0}$ to the "dressed" one: $T_{2}^{-1} L$; see (2.38). Thus, $\nu$ is a transformation (dressing) operator. Explicitly, using (2.24) and (2.38), we see that equation (2.37) reads as

(2.41) $\nu\left(m+e_{2}\right)\left(T_{1}+a_{1}-a_{2}\right)=\left(T_{1}+a_{1}-a_{2}\right) \nu(m)+\left[U\left(m+e_{2}\right)-U\left(m+e_{1}\right)\right] \nu(m)$,

which is the first equation of the Lax pair. The second equation of the Lax pair can be deduced similarly if we notice that, by (2.19) with $n=3$ and (2.22), we have $B\left(m+e_{3}\right)=$ $\left(T_{1}+a_{1}-a_{3}\right) B(m)\left(T_{1}+a_{1}-a_{3}\right)^{-1}$. Then as above we get

$$
\nu\left(m+e_{3}\right)\left(T_{1}+a_{1}-a_{3}\right)=\left(T_{1}+a_{1}-a_{3}\right) \nu(m)+\left[U\left(m_{3}+1\right)-U\left(m_{1}+1\right)\right] \nu(m) .
$$

In order to present these equations in a more standard form, we introduce the Jost solution via the kernel of the transformation operator

$$
\varphi\left(m, z_{1}\right)=z_{1}^{m_{1}}\left(z_{1}+a_{1}-a_{2}\right)^{m_{2}}\left(z_{1}+a_{1}-a_{3}\right)^{m_{3}} \oint_{|\zeta|=1}\left(\frac{d \zeta}{2 \pi i \zeta}\right)^{2} \nu(m, \zeta ; z),
$$

so that $z_{1}$ plays the role of the spectral parameter. Then (2.41) takes the form

$$
\varphi\left(m+e_{2}, k\right)=\varphi\left(m+e_{1}, k\right)+\left(u\left(m+e_{2}\right)-u\left(m+e_{1}\right)+a_{1}-a_{2}\right) \varphi(m, k),
$$

and (2.42) reads as

$$
\varphi\left(m+e_{3}, k\right)=\varphi\left(m+e_{1}, k\right)+\left(u\left(m+e_{3}\right)-u\left(m+e_{1}\right)+a_{1}-a_{3}\right) \varphi(m, k),
$$

where the spectral parameter is denoted by $k=z_{1}$ for simplicity. We mention that, by (2.32) and (2.43), the Jost solution is normalized at $k$-infinity, $k \in \mathbb{C}$, as

$$
\lim _{k \rightarrow \infty} k^{-m_{1}}\left(k+a_{1}-a_{2}\right)^{-m_{2}}\left(k+a_{1}-a_{3}\right)^{-m_{3}} \varphi(m, k)=1 .
$$

We see that, by the symmetry of the problem, the first and second equations of the Lax pair can be exchanged. Observe that the difference of these equations

$$
\varphi\left(m+e_{3}, k\right)=\varphi\left(m+e_{2}, k\right)+\left(u\left(m+e_{3}\right)-u\left(m+e_{2}\right)+a_{2}-a_{3}\right) \varphi(m, k)
$$

is also symmetric with respect to the previous equations, so that any two of them can be taken as the Lax pair.

The compatibility condition of these two equations gives

$$
\begin{aligned}
{\left[a_{1}-a_{2}-\Delta_{1} u+\Delta_{2} u\right] \Delta_{1} \Delta_{2} u } & +\left[a_{2}-a_{3}-\Delta_{2} u+\Delta_{3} u\right] \Delta_{2} \Delta_{3} u \\
+ & {\left[a_{3}-a_{1}-\Delta_{3} u+\Delta_{1} u\right] \Delta_{3} \Delta_{1} u=0, }
\end{aligned}
$$

where $\Delta$ is the difference operator defined in (1.5); this is the Hirota difference equation for the function $u=u\left(m_{1}, m_{2}, m_{3}\right)$. Clearly, equation (1.8) is a linearization of this equation.

\subsection{Some remarks on the Hirota difference equation.}

2.4.1. The Hirota difference equation was introduced in [12, 13] in its bilinear form, the Hirota bilinear difference equation (HBDE), as an equation on the tau-function. It is often regarded as a fundamental integrable system, because many other integrable equations, such as KdV, KP, mKdV, mKP, the two-dimensional Toda lattice, the sine-Gordon, the Benjamin-Ono, etc., were shown to arise as special (continuous) limits of the HBDE. It also presents itself as a model-independent functional relation for the eigenvalues of quantum transfer matrices. A detailed survey of this equation was given in [14]; see also the references therein. Elliptic solutions of the HBDE were considered in [15]. In [16] it was called the "generalized KP hierarchy", and equation (4) of that article can be rewritten in the form (2.48) after the change of parameters $a_{j} \mapsto-1 / a_{j}$. 
2.4.2. In Subsection 1.1 we mentioned that the case where at least two parameters $a_{n}$ coincide is degenerate. The same can be seen from (2.48). Say, if $a_{1}=a_{2}$, then equation (2.48) has a solution $u$ such that $\Delta_{1} u=\Delta_{2} u$. Thus, the condition that all the $a_{n}$ are different is essential to have a nontrivial equation on $u_{m}$. But even under this condition, if the equation $\Delta_{1} u=\Delta_{2} u$ is valid, then (2.48) reduces to the linear equation $\left[\left(a_{1}-a_{2}\right) \Delta_{1} u+\left(a_{2}-a_{3}\right) \Delta_{3}\right] \Delta_{1} u=0$ again. Thus, it is natural to assume that all finite differences $\Delta_{n} u$ are distinct, at least at the initial value of $m_{3}$. On the other hand, it is clear that, introducing the new dependent variable

$$
v(m)=u(m)-a_{1} m_{1}-a_{2} m_{2}-a_{3} m_{3},
$$

we can remove the parameters $a_{n}$ from equations (2.48) and (2.44), (2.45) (or (2.47) ) of the Lax pair, e.g.,

$$
\left(-v_{1}+v_{2}\right) v_{1,2}+\left(-v_{2}+v_{3}\right) v_{2,3}+\left(-v_{3}+v_{1}\right) v_{3,1}=0 .
$$

Since these parameters play an essential role in the evolution (2.29) of the spectral data, this means that the direct (see $\$ 3$ ) and inverse problems are solvable only in the case where $u(m)$ decays sufficiently rapidly as $m \rightarrow \infty$. The condition that $u(m)$ decays at least in some direction is necessary for uniqueness: addition of a constant to $u(m)$ affects neither equation (2.48), nor the Lax pair.

2.4.3. The relationship between (2.48) and the $\tau$-function formulation of the HBDE follows from [17; in our notation here it looks like this:

$$
\begin{aligned}
& v\left(m+e_{1}\right)-v\left(m+e_{3}\right)=\frac{\tau\left(m+e_{1}+e_{3}\right) \tau(m)}{\tau\left(m+e_{1}\right) \tau\left(m+e_{3}\right)}, \\
& v\left(m+e_{2}\right)-v\left(m+e_{1}\right)=\frac{\tau\left(m+e_{2}+e_{1}\right) \tau(m)}{\tau\left(m+e_{2}\right) \tau\left(m+e_{1}\right)},
\end{aligned}
$$

where the two equations are related by a cyclic permutation of $\{1,2,3\}$. The third equation

$$
v\left(m+e_{3}\right)-v\left(m+e_{2}\right)=\frac{\tau\left(m+e_{3}+e_{2}\right) \tau(m)}{\tau\left(m+e_{3}\right) \tau\left(m+e_{2}\right)},
$$

which can also be obtained by cyclic permutation follows from the identity

$$
\begin{aligned}
\tau\left(m+e_{1}\right) \tau\left(m+e_{2}+e_{3}\right) & +\tau\left(m+e_{2}\right) \tau\left(m+e_{3}+e_{1}\right) \\
& +\tau\left(m+e_{3}\right) \tau\left(m+e_{1}+e_{2}\right)=0 .
\end{aligned}
$$

Precisely this relation is called the Hirota bilinear discrete equation; note that, as in the case of other discrete systems, it can be written in fairly different forms (see [14]).

\section{§3. Direct PRoblem}

Here we briefly discuss the direct problem for the Lax operator (2.38). In the framework of the extended resolvent approach (see, say, 4] for details) the main object of the construction is the resolvent, i.e., the inverse of the operator $L$ in the sense of composition (2.7): $L M=I=M L$. Under the conditions on $U$ formulated in Subsection 2.4, we can define the resolvent with the help of the equation $M=M_{0}+M_{0}\left(\Delta_{2} U-\Delta_{1} U\right) M$ or its dual, where

$$
M_{0}(\zeta ; z)=\frac{\delta_{c}(\zeta)}{z_{2}-z_{1}+a_{2}-a_{1}}
$$


is the resolvent corresponding to the case of the zero potential $U$, i.e., the inversion of the operator $L_{0}$ in (2.24). Explicitly, we get the following integral equation for $M$ :

$$
M(\zeta ; z)=\frac{\delta_{c}(\zeta)}{z_{2}-z_{1}+a_{2}-a_{1}}+\frac{\left(\left(\Delta_{2} U-\Delta_{1} U\right) M\right)(\zeta ; z)}{z_{2} \zeta_{2}-z_{1} \zeta_{1}+a_{2}-a_{1}} .
$$

By definition, the resolvent obeys the Hilbert identity $M^{\prime}-M=-M^{\prime}\left(L_{0}^{\prime}-L_{0}\right) M$, where $L^{\prime}$ and $M^{\prime}$ have kernels $L(\zeta ; z w)$ and $M(\zeta ; z w)$, respectively, and $w$ is a complex parameter. Then, using (2.16) and (2.17), for the $\bar{\partial}$-derivatives of $M$ we get

$$
\bar{\partial}_{j} M=\nu\left(\bar{\partial}_{j} M_{0}\right) T_{j}^{-1} \omega T_{j}, \quad j=1,2 .
$$

Here $\nu$ and $\omega$ are operators with the kernels

$$
\begin{aligned}
& \nu(\zeta ; z)=\left.\left(M L_{0}\right)(\zeta ; z)\right|_{z_{2}=z_{1}+a_{1}-a_{2}}, \\
& \omega(\zeta ; z)=\left.\left(L_{0} M\right)(\zeta ; z)\right|_{z_{2}=\left(z_{1} \zeta_{1}+a_{1}-a_{2}\right) \bar{\zeta}_{2}},
\end{aligned}
$$

where reduction appeared thanks to the $\bar{\partial}$-derivative of (3.1). The operator $\nu$ can also be defined by the following integral equation, which follows from (3.2) and (3.4):

$$
\nu(\zeta ; z)=\delta_{c}(\zeta)+\frac{\left(\left(\Delta_{2} U-\Delta_{1} U\right) \nu\right)(\zeta ; z)}{z_{1}\left(\zeta_{2}-\zeta_{1}\right)+\left(a_{2}-a_{1}\right)\left(1-\zeta_{2}\right)} .
$$

It is easy to check that this $\nu$ satisfies equation (2.37) and the asymptotic condition (2.36), so that it coincides with the transformation (dressing) operator introduced in Subsection 2.3. Leaving details for a forthcoming publication, we only mention that $\omega$ is the dual transformation operator. In Subsection 2.3. the dressing operator $\nu$ was defined by equation (2.30), i.e., the equation of the inverse problem. Here, in the direct problem, we also get relation (2.28), where

$$
b(\zeta)=\left.\pi^{2} \frac{\left(\left(\Delta_{2} U-\Delta_{1} U\right) \nu\right)\left(\zeta ; z_{1}\right)}{\zeta_{2}-\zeta_{1}}\right|_{z_{1=\left(a_{2}-a_{1}\right)\left(\zeta_{2}-1\right) /\left(\zeta_{1}-\zeta_{2}\right)}},
$$

which gives an expression for the scattering data $b(\zeta)$ in terms of the potential and transformation operator (i.e., the Jost solution if we use (2.43)).

\section{$\S 4$. Discussion}

In the present paper, the observation of 1 and 2 concerning the equivalence between the integrability of nonlinear equations and the existence of commutator identities on associative algebras was carried over to the case of purely discrete systems. On the other hand, some identities used in 1 and 2 can be obtained as special limits of (1.3). Indeed, we have $\lim _{a \rightarrow \infty} a \delta_{a}(B)=-[A, B]$ by (1.2). Thus, in the limit as $a_{3} \rightarrow \infty$, equation (1.3) yields the identity

$$
\left[A, \delta_{a_{1}}(B)-\delta_{a_{2}}(B)\right]+\left(a_{2}-a_{1}\right) \delta_{a_{1}} \delta_{a_{2}}(B)=0
$$

which involves both types of commutators. By analogy with (1.4), we introduce two discrete "times" $m_{1}, m_{2}$ and a continuous time $t$ :

$$
B\left(m_{1}, m_{2}, t\right)=\left(\prod_{n=1}^{2}\left(A-a_{n}\right)^{m_{n}} e^{t A}\right) B\left(\prod_{n=1}^{2}\left(A-a_{n}\right)^{-m_{n}} e^{-t A}\right) .
$$

This function solves the linear differential-difference equation

$$
\left[\partial_{t}\left(\Delta_{1}-\Delta_{2}\right)+\left(a_{2}-a_{1}\right) \Delta_{1} \Delta_{2}\right] B\left(m_{1}, m_{2}, t\right)=0 .
$$

The dressing procedure for this case can be developed as was explained in 2, leading to the corresponding nonlinear integrable equation and its Lax pair. 
At the next step of reduction, we can put $a_{1}=0$ in (4.1), divide by $a_{2}$, and consider the limit as $a_{2} \rightarrow 0$. This gives the identity

$$
\left[A,\left[A^{-1}, B\right]\right]=2 B-A B A^{-1}-A^{-1} B A,
$$

again involving commutators of both types. Introducing

$$
B\left(m_{1}, t_{2}, t_{3}\right)=\left(A^{m_{1}} e^{t_{2} A+t_{3} A^{-1}}\right) B\left(A^{m_{1}} e^{t_{2} A+t_{3} A^{-1}}\right)^{-1},
$$

we see that this function obeys the differential-difference equation

$$
\partial_{t_{2}} \partial_{t_{3}} B\left(m_{1}, t_{2}, t_{3}\right)=2 B\left(m_{1}, t_{2}, t_{3}\right)-B\left(m_{1}-1, t_{2}, t_{3}\right)-B\left(m_{1}+1, t_{2}, t_{3}\right),
$$

which is a linearized version of the Toda chain

$$
\partial_{t} \partial_{x} \phi_{m}=e^{\phi_{m}-\phi_{m-1}}-e^{\phi_{m+1}-\phi_{m}} .
$$

Identity (4.4) was considered in [2, where the dressing procedure for recovering equation (4.7) with the help of identity (4.4) was demonstrated.

Finally, we perform the substitution $A \rightarrow e^{\alpha A}$ in (4.4) and take the fourth derivative of the resulting identity with respect to $\alpha$ at zero. This results in the commutator identity

$$
4\left[A^{3},[A, B]\right]-3\left[A^{2},\left[A^{2}, B\right]\right]-[A,[A,[A,[A, B]]]]=0,
$$

which was the initial identity in [1]. Here $A$ and $B$ can be arbitrary elements of an arbitrary associative algebra. Now we define a function of the times $t_{n} \in \mathbb{R}, n=1,2,3$, as follows:

$$
B\left(t_{1}, t_{2}, t_{3}\right)=e^{t_{1} A+t_{2} A^{2}+t_{3} A^{3}} B e^{-t_{1} A-t_{2} A^{2}-t_{3} A^{3}} ;
$$

this function satisfies a linearized version of the Kadomtsev-Petviashvili equation. See [1] for a detailed discussion of the construction based on (4.8) and (4.9).

Our approach, based on the commutator identities, is close to Manakov's observation [18] of the relationship between integrability and the degenerate dispersion law, although in our case the inverse problem is derived, not assumed. Moreover, the method of identities is applicable to a wider class of integrable equations. Suppose that, besides $A$ and $B$, we have another element $\sigma$ of the same associative algebra that obeys

$$
[A, \sigma]=0, \quad\{B, \sigma\}=0, \quad \sigma^{2}=1,
$$

where $\{$,$\} denotes the anticommutator. (For example, A$ and $B$ are diagonal and offdiagonal $2 \times 2$ matrices with elements belonging to an associative algebra, and $\sigma=\sigma_{3}$, the Pauli matrix.) Then we have

$$
[A \sigma, B]=\sigma\{A, B\}
$$

which extends the set of commutator identities essentially. For example, the identity $\left[A^{2}, B\right]=[A,\{A, B\}]$ can be written in the commutator form as

$$
\sigma\left[A^{2}, B\right]=[A,[A \sigma, B]] .
$$

Introducing a dependence on three times by

$$
B\left(t_{1}, t_{2}, t_{3}\right)=e^{t_{1} A+t_{2} A \sigma+t_{3} A^{2}} B e^{-t_{1} A-t_{2} A \sigma-t_{3} A^{2}},
$$

we get a linearized version of the DS equation:

$$
\left(\sigma \partial_{t_{3}}-\partial_{t_{1}} \partial_{t_{2}}\right) B(t)=0 .
$$

Other kinds of commutator identities can be found in [2]. 


\section{REFERENCES}

[1] A. K. Pogrebkov, Commutator identities on associative algebras and the integrability of nonlinear evolution equations, Teoret. Mat. Fiz. 154 (2008), no. 3, 477-491; English transl., Theoret. and Math. Phys. 154 (2008), 405-417. MR2431558 (2009f:37072)

[2] 2D Toda chain and associated commutator identity, Geometry, Topology, and Mathematical Physics, Amer. Math. Soc. Transl. Ser. 2, vol. 224, Amer. Math. Soc., Providence, RI, 2008, pp. 261-269. MR2462365 (2010a:37155)

[3] M. Boiti, F. Pempinelli, A. K. Pogrebkov, and M. C. Polivanov, Resolvent approach for twodimensional scattering problems. Application to the nonstationary Schrödinger problem and the KPI equation, Teoret. Mat. Fiz. 93 (1992), no. 2, 181-210; English transl., Theoret. and Math. Phys. 93 (1992), no. 2, 1200-1224 (1993). MR1233541 (95e:35193)

[4] _ Resolvent approach for the nonstationary Schrödinger equation, Inverse Problems 8 (1992), 331-364. MR1166486 (93m:35159)

[5] M. Boiti, F. Pempinelli, and A. Pogrebkov, Properties of solutions of the Kadomtsev-Petviashvili I equation, J. Math. Phys. 35 (1994), 4683-4718. MR1290895 (95f:35217)

[6] M. Boiti, F. Pempinelli, A. K. Pogrebkov, and B. Prinari, Extended resolvent and inverse scattering with an application to KPI, J. Math. Phys. 44 (2003), 3309-3340. MR2006753 (2004j:37137)

[7] E. Date, M. Jimbo, M. Kashiwara, and T. Miwa, Transformation groups for soliton equations, Nonlinear Integrable Systems: Classical Theory and Quantum Theory (Kyoto, 1981), World Sci., Singapore, 1983, pp. 39-119. MR0725700 (86a:58093)

[8] A. Yu. Orlov and E. I. Schulman, Additional symmetries for integrable equations and conformal algebra representation, Lett. Math. Phys. 12 (1986), 171-179. MR0865754 (88g:58085)

[9] A. Yu. Orlov, Vertex operator, $\bar{\partial}$-problem, symmetries, variational identities, and Hamiltonian formalism for $(2+1)$ integrable systems, Plasma Theory and Nonlinear and Turbulent Processes in Physics (Kiev, 1987), Vol. 1, World Sci., Singapore, 1988, pp. 116-134. MR0957158 (89k:58128)

[10] V. E. Zakharov and A. B. Shabat, A plan for integrating the nonlinear equations of mathematical physics by the method of the inverse scattering problem. I, Funktsional. Anal. i Prilozhen. 8 (1974), no. 3, 43-53; English transl., J. Funct. Anal. Appl. 8 (1974), no. 2, 226-235. MR0481668 (58:1768)

[11] A. K. Pogrebkov, On time evolutions associated with the nonstationary Schrödinger equation, L. D. Faddeev's Seminar on Mathematical Physics, Amer. Math. Soc. Transl. (2), vol. 201, Amer. Math. Soc., Providence, RI, 2000, pp. 239-255. MR.1772293 (2001f:35100)

[12] R. Hirota, Nonlinear partial difference equations. II. Discrete time Toda equation, J. Phys. Soc. Japan 43 (1977), 2074-2078. MR0460935 (57:925b)

[13] _ Discrete analogue of a generalized Toda equation, J. Phys. Soc. Japan 50 (1981), 37853791. MR0638804 (83e:58035)

[14] A. Zabrodin, A survey of Hirota's difference equations, solv-int/9704001 (1997). MR1608967 (2000d:39009)

[15] I. Krichever, P. Wiegmann, and A. Zabrodin, Elliptic solutions to difference non-linear equations and related many-body problems, hep-th/9704090 (1997). MR1618143 (99e:58103)

[16] L. V. Bogdanov and B. G. Konopelchenko, Generalized KP hierarchy: Möbius symmetry, symmetry constraints and Calogero-Moser system, solv-int/9912005 (1999).

[17] A. Zabrodin, Bäcklund transformations and Hirota equation and supersymmetric Bethe ansatz, arXiv: 0705.4006v1 (2007).

[18] S. V. Manakov, The inverse scattering transform for the time-dependent Schrödinger equation and Kadomtsev-Petviashvili equation, Physica D 3 (1981), 420-427.

Steklov Mathematical Institute, Moscow 119991, Russia

E-mail address: pogreb@mi.ras.ru

Received 19/FEB/2010

Originally published in English 